\title{
COMPARACIÓN DE SORGOS (Sorghum bicolor L.) ISOGÉNICOS PARA DOS TIPOS DE CITOPLASMA, APTITUD COMBINATORIA GENERAL Y ESPECÍFICA ${ }^{1}$
}

\author{
Héctor Williams ${ }^{2}$, Raúl Rodríguez ${ }^{2}$
}

\begin{abstract}
RESUMEN
Comparación de sorgos (Sorghum bicolor L.) isogénicos para dos tipos de citoplasma, aptitud combinatoria general y específica. El objetivo de este estudio fue comparar las estimaciones de aptitud combinatoria en un grupo de híbridos isogénicos para tipo de citoplasma $\left(\mathrm{A}_{1} \mathrm{y} \mathrm{A}_{2}\right)$, estudiando la posibilidad de utilizar el sistema $\mathrm{A}_{2}$ a nivel comercial. Los genotipos se evaluaron en diez ambientes y se tomaron datos de rendimiento de grano, días a floración, porcentaje de humedad del grano, altura de planta, longitud de panícula y longitud de excerción. Los cálculos para estimar aptitud combinatoria general (ACG) y específica (ACE) se hicieron de acuerdo al modelo propuesto por Beil y Atkins en 1967. Los resultados indicaron que aunque en general los mejores valores de ACG y ACE correspondieron a genotipos con el citoplasma $\mathrm{A}_{2}$, no se encontró relación con el tipo de citoplasma. También sugiere que el citoplasma $\mathrm{A}_{2}$ puede ser usado para la produc-ción comercial de híbridos de sorgo como una alternativa al citoplasma $\mathrm{A}_{1}$.
\end{abstract}

\begin{abstract}
Comparison of isogenic sorghums for two types of cytoplasm and general and specific combining aptitude. The purpose of this study was to compare estimates of relati ve general and specific combining ability effects on isogenic hybrids in two different cytoplasm types $\left(\mathrm{A}_{1}\right.$ and $\left.\mathrm{A}_{2}\right)$, and observe the possibility of use the $\mathrm{A}_{2}$ system for the commercial production of sorghum hybrids. Genotypes were evaluated in ten environments located in northern Mexico. Grain yield, days to flowering, grain moisture content at harvest, plant height, panicle lenght and panicle excertion were recorded from the experiments. Combining ability was estimated ac-cording to the model suggested by Beil and Atkins in 1967. Although in all studied, the characteristics generally, the best SCA and GCA were for $\mathrm{A}_{2}$ cytoplasm, there was no relation between cytoplasm type and combining ability. These results indicate that $\mathrm{A}_{2}$ cytoplasm can be used for commercial hybrid sorghum seed production, as an alternative to $\mathrm{Al}$ cytoplasm.
\end{abstract}

\section{INTRODUCCIÓN}

El descubrimiento de la esterilidad génico citoplásmica en sorgo o millo Sorghum bicolor (L) Moench, (Stephens y Holland, 1954), en citoplasma de milo y genes nucleares de Kafir, abrió el camino a la utilización de híbridos de sorgo a nivel comercial (Schertz y Ritchey, 1978).

Debido a que en sorgo a nivel comercial se utiliza un solo sistema de androesterilidad génico-citoplásmica para formar los híbridos, es necesario investigar otras fuentes de androesterilidad (Ganga y Borikar, 1989; Worstell, Kidd y Schertz, 1984; Schertz y Ritchey, 1978; Ross y Kofoid, 1979). El mismo problema se indica en maíz y "mijo perla" (Hanna, 1989).

Nuevos sistemas de androesterilidad génico citoplásmica han sido descubiertos en sorgo, los cuales pueden aumentar la variabilidad y reducir los problemas de vulnerabilidad genética. La fuente de androesterilidad génico-citoplásmica $\mathrm{A}_{2}$, está representada por la línea IS$12662 \mathrm{C}$, perteneciente al grupo Caudatum nigrians de la raza Guinea originaria de Etiopía. La fuente de genes

1 Presentado en la XL Reunión Anual del PCCMCA en Costa Rica, América Central. 13 al 19 de marzo, 1994.

2 Investigadores del Programa de Cultivos Industriales. Subprograma Sorgo del Instituto Nacional de Investigaciones Forestales y Agropecuarias (INIF AP). Campo Agrícola Experimental de Río Bravo, Apdo. Postal 172. Río Bravo. Tam. c.P. 88900 México. 
nucleares se encontró en la línea IS5322C (SC-250), del grupo Roxburghi de la raza Guinea originaria de la India (Schertz, 1977).

La presencia de información genética en los organelos del citoplasma, sugiere para la mejor expresión de ciertas características, la posibilidad de una combinación óptima entre el núcleo y el citoplasma (Lee, Albertsen y Rasmusson, 1987). Por otra parte, no conocemos si algunos de estos citoplasmas tienen diferentes respuestas a un problema. Se han enfocado los esfuerzos a vulnerabilidad hacia una enfermedad, sin embargo otro tipo de problemas v.g. fisiológicos no se deben descuidar (Ross y Kofoid, 1979). El objetivo de este estudio fue comparar las estimaciones de aptitud combinatoria general (ACG) y aptitud combinatoria específica (ACE), en un grupo de híbridos isogénicos para dos tipos de citoplasma $\left(\mathrm{A}_{1} \mathrm{y} \mathrm{A}_{2}\right)$.

\section{MATERIALES Y MÉTODOS}

\section{Genotipos}

Se evaluaron 32 híbridos isogénicos $\left(16 \mathrm{~A}_{1}\right.$ y $\left.16 \mathrm{~A}_{2}\right)$ para el tipo de androesterilidad génico-citoplásmica, formados al hacer todas las combinaciones posibles con las siguientes líneas progenitoras: Líneas hembras isogénicas para tipo de citoplasma $\left(4 \mathrm{~A}_{1} \mathrm{y}_{4} \mathrm{~A}_{2}\right)$; LRB102A, LRB-104A, LRB-106A y LRB-110A y como líneas R (machos); LRB-25, LRB-63, SC0 599-11E y 74CS-5388. Las líneas LRB son genotipos mexicanos obtenidos en el Campo Experimental Río Bravo, mientras que las otras líneas fueron introducidas de Texas, E.U.A. La fuente de androesterilidad $\mathrm{A}_{2}$ fue llevada a Río Bravo en 1982, y se empezó a introducir a las líneas $\mathrm{A}_{1}$ en el siguiente año, hasta que contaron con ocho retrocruzas hacia $\mathrm{A}_{2}$. La semilla de los híbridos fue producida mediante polinizaciones controladas a mano.

\section{Localidades}

Los híbridos fueron evaluados en diez ambientes (cinco de riego y cinco de temporal) localizados en la región norte de México. Bajo condiciones de riego los experimentos fueron sembrados en Río Bravo, Tam. en 1990, 1991 (en dos fechas de siembra) y en 1992, y en los Mochis, Sin. en 1991. En tanto que en temporal fueron en los siguientes ambientes localizados en el estado de Tamaulipas; 1991 en Díaz Bazan y El Tapón, y en 1992 en El Tapón, El Canelo y rancho Guelatao.

\section{Diseño experimental}

En cada localidad se utilizó un diseño de látice triple con tres repeticiones, con parcelas de $5 \mathrm{~m}$ de largo y 0,80 $\mathrm{m}$ de separación. El manejo de cada experimento se realizó de acuerdo a las recomendaciones regionales de cada localidad en cuanto a fechas de siembra, densidad de población, fertilización,. cultivos, riegos, control de plagas, etc.

\section{Datos tomados}

Para cada experimento fueron rendimiento de grano, días a floración, porcentaje de humedad del grano a la cosecha, altura de planta (m), longitud de panícula y excerción $(\mathrm{cm})$.

\section{Análisis estadístico}

Para estimar la aptitud combinatoria general (ACG) y aptitud combinatoria específica (ACE) en todas las características estudiadas, y en diez ambientes, se utilizó el modelo propuesto por Beil y Atkins en 1967. El análisis de varianza para calcular los efectos genéticos cuantitativos se hizo de acuerdo al modelo que se presenta en el Cuadro 1. Los valores así obtenidos de ACG y ACE se relacionaron entre los híbridos isogénicos para los dos tipos de citoplasma.

\section{RESULTADOS Y DISCUSION}

Las medias de las características agronómicas de los híbridos para los dos tipos de citoplasma, se muestran en el Cuadro 2, donde se observa que son numéricamente muy parecidos. Williams y Rodríguez (1994), al estudiar 
Cuadro 1. Análisis de varianza utilizado para calcular los efectos genéticos cuantitativos.

\begin{tabular}{lcl}
\hline Causas Variación & G.L. & Esperanzas de Cuadrados Medios \\
\hline Machos & 3 & $\sigma^{2} \mathrm{e}+\mathrm{h} \sigma^{2} \mathrm{a}+\mathrm{r} \sigma^{2} \mathrm{lhm}+\mathrm{rh} \sigma^{2} \mathrm{ml}+\mathrm{rl} \sigma^{2} \mathrm{hm}+\mathrm{rlh} \sigma^{2} \mathrm{~m}$ \\
MXA & 27 & $\sigma^{2} \mathrm{e}+\mathrm{h} \sigma^{2} \mathrm{a}+\mathrm{r} \sigma^{2} \mathrm{lhm}+\mathrm{rhh} \sigma^{2} \mathrm{ml}$ \\
MXR Error (a) & 60 & $\sigma^{2} \mathrm{e}+\mathrm{h} \sigma^{2} \mathrm{a}$ \\
Hembras & 7 & $\sigma^{2} \mathrm{e}+\mathrm{r} \sigma^{2} \mathrm{lhm}+\mathrm{rm} \sigma^{2} \mathrm{hl}+\mathrm{rl} \sigma^{2} \mathrm{hm}+\mathrm{rlm} \sigma^{2} \mathrm{~h}$ \\
HXA & 63 & $\sigma^{2}+\mathrm{r} \sigma^{2} \mathrm{hm}+\mathrm{rm} \sigma^{2} \mathrm{hl}$ \\
HXM & 21 & $\sigma^{2} \mathrm{e}+\mathrm{r} \sigma^{2} \mathrm{hm}+\mathrm{rl} \sigma^{2} \mathrm{hm}$ \\
HXMXA & 189 & $\sigma^{2} \mathrm{e}+\mathrm{r} \sigma^{2} \mathrm{lhm}$ \\
HXMXR Error (b) & 420 & $\sigma^{2} \mathrm{e}$ \\
\hline
\end{tabular}

Cuadro 2. Medias de los híbridos F1 en dos tipos de citoplasma y con una hembra en común.

\begin{tabular}{|c|c|c|c|c|c|c|}
\hline Genealogía & $\begin{array}{l}\text { Rend. } \\
\text { kg/ha }\end{array}$ & $\begin{array}{c}\text { Días a } \\
\text { Flor }\end{array}$ & $\begin{array}{l}\text { Altura } \\
\text { PIta.m }\end{array}$ & $\begin{array}{l}\text { \% Humedad } \\
\text { Grano }\end{array}$ & $\begin{array}{c}\text { Longitud } \\
\text { Panícula } \\
(\mathbf{c m})\end{array}$ & $\begin{array}{c}\text { Longitud } \\
\text { Excerción } \\
(\mathbf{c m})\end{array}$ \\
\hline $\begin{array}{l}\text { LRB-104A } \\
\text { LRB-104A } \\
\text { LRB-106A } \\
\text { LRB-106A } \\
\text { LRB-102A } \\
\text { LRB-102A } \\
\text { LRB-110A } \\
\text { LRB-110A }\end{array}$ & $\begin{array}{l}4261 \\
3991 \\
4103 \\
4303 \\
4186 \\
4189 \\
4028 \\
4140\end{array}$ & $\begin{array}{l}78,0 \\
76,7 \\
74,2 \\
78,1 \\
76,7 \\
76,6 \\
76,3 \\
76,1\end{array}$ & $\begin{array}{l}1,40 \\
1,40 \\
1,39 \\
1,47 \\
1,46 \\
1,45 \\
1,40 \\
1,36\end{array}$ & $\begin{array}{l}15,28 \\
15,22 \\
14,40 \\
14,63 \\
14,79 \\
14,83 \\
15,04 \\
15,03\end{array}$ & $\begin{array}{l}31,3 \\
31,5 \\
28,5 \\
29,3 \\
31,4 \\
30,0 \\
29,7 \\
30,3\end{array}$ & $\begin{array}{l}15,2 \\
14,6 \\
17,0 \\
16,3 \\
16,8 \\
18,4 \\
19,3 \\
19,1\end{array}$ \\
\hline $\begin{array}{l}\mathrm{X} \text { hembras } \mathrm{A}_{1} \\
\mathrm{X} \text { hembras } \mathrm{A}_{2}\end{array}$ & $\begin{array}{l}4145 \\
4156 \\
\end{array}$ & $\begin{array}{l}76,3 \\
76,9 \\
\end{array}$ & $\begin{array}{l}1,41 \\
1,42 \\
\end{array}$ & $\begin{array}{l}14,88 \\
14,93 \\
\end{array}$ & $\begin{array}{l}30,2 \\
30,3 \\
\end{array}$ & $\begin{array}{l}17,01 \\
17,10 \\
\end{array}$ \\
\hline
\end{tabular}

los mismos genotipos encontraron que el tipo de citoplasma, no afectó significativamente estas características agronómicas, excepto para días a floración donde en condiciones de riego los híbridos A2 florecieron un día mas tarde que los híbridos A,.

En los Cuadros 3 y 4 encontramos las estimaciones de aptitud combinatotia general y específica de los híbridos $F_{1}$ para las características de rendimiento de grano y días a floración. Los resultados de ACG para las siete características estudiadas indicaron que para los caracteres de rendimiento de grano, días a floración y altura de planta, la mejor ACG para las líneas hembras fue para LRB-106A 2 ; para porcentaje de humedad del grano y longitud de panoja, correspondió paraLRB-104A $\mathrm{A}_{1}$ y para longitud de excerción para LRB- $110 \mathrm{~A}_{1}$ y $\mathrm{A}_{2}$.
Respecto a las líneas R el mejor valor de ACG para días a floración, altura de planta, porcentaje de humedad del grano y longitud de panícula correspondieron a la línea LRB-25. En tanto que para rendimiento de grano y longitud de excerción, correspondieron los valores más altos para SCO 599-11 E. Se conoce que las líneas que presentan una ACG alta, son buenas combinadoras y es de esperarse que se comporten bien con la mayoría de los híbridos en donde intervienen como progenitores.

En cuanto a la ACE las mejores combinaciones híbridas fueron respectivamente para rendimiento de grano LRB-110A 2 x74CS-5388 y LRB-110A 2 xLRB-25 (Cuadro 3), para días a floración LRB- $104 \mathrm{~A}_{2} \times \mathrm{LRB}-25$ (Cuadro 4), para altura de planta LRB-102 $\mathrm{A}_{1} \times \mathrm{LRB}-25$ $(0,03)$ y LRB-110A 2 x 74CS-5388 $(0,03)$, para porcentaje 
Cuadro 3. Estimación de los efectos de aptitud combinatoria general y específica para rendimiento de grano, basado en los datos de los híbridos isogénicos F1 (A1 y A2) evaluados en diez ambientes.

\begin{tabular}{|c|c|c|c|c|c|}
\hline \multirow[t]{3}{*}{ Hembras } & \multicolumn{4}{|c|}{ Machos } & \multirow{3}{*}{$\begin{array}{l}\text { Efectos } \\
\text { Generales } \\
\text { Hembra }\end{array}$} \\
\hline & \multirow[b]{2}{*}{ LRB-63 } & \multicolumn{2}{|c|}{ Efectos específicos } & \multirow{2}{*}{ SCO 599-11E } & \\
\hline & & LRB-25 & $74 C S-5388$ & & \\
\hline LRB-104A1 & 0 & 0,15 & 0,03 & 0,12 & 0,03 \\
\hline LRB-104A2 & 0,08 & 0,06 & 0,03 & 0,04 & 0,04 \\
\hline LRB-106A1 & 0,04 & 0,08 & 0,15 & 0,3 & 0,01 \\
\hline LRB-106A2 & 0,03 & 0,03 & 0,05 & 0,05 & 0,04 \\
\hline LRB-102A1 & 0,05 & 0,09 & 0 & 0,04 & 0,01 \\
\hline LRB-102A2 & 0,04 & 0,14 & 0,01 & 0,05 & 0,01 \\
\hline LRB-110A1 & 0,06 & 0,01 & 0,09 & 0,01 & 0,03 \\
\hline LRB-110A2 & 0,12 & 0,04 & 0,14 & 0,03 & 0 \\
\hline Efectos Grales. de machos & 0,03 & 0 & 0,01 & 0,04 & \\
\hline
\end{tabular}

Cuadro 4. Estimación de los efectos de aptitud combinatoria general y específica para días a floración, basadas en los datos de los híbridos isogénicos F1 (A1 y A2) evaluados en diez ambientes.

\begin{tabular}{lccccc}
\hline Hembras & \multicolumn{3}{c}{ Machos } & \multirow{2}{*}{$\begin{array}{c}\text { Efectos } \\
\text { Generales } \\
\text { Hembra }\end{array}$} \\
\cline { 2 - 5 } & LRB-63 & LRB-25 & $\mathbf{7 4 C S - 5 3 8 8}$ & SCO 599-11E & 0,02 \\
\cline { 2 - 5 } LRB-104 A1 & 0,01 & 0,02 & 0,01 & 0 & 0 \\
LRB-104 A2 & 0,01 & 0,05 & 0,04 & 0 & 0,03 \\
LRB-106 A1 & 0 & 0,01 & 0,01 & 0,01 & 0,02 \\
LRB-106 A2 & 0,01 & 0,01 & 0 & 0,01 & 0 \\
LRB-102 A1 & 0,01 & 0 & 0,01 & 0 & 0,01 \\
LRB-102 A2 & 0,01 & 0 & 0 & 0 & 0 \\
LRB-110 A1 & 0,01 & 0,01 & 0,01 & 0 & 0 \\
LRB-110 A2 & 0,01 & 0,04 & 0,03 & 0,01 & \\
Efecto Gral. de Machos & 0,01 & 0,04 & 0,03 & 0,03 & \\
\hline
\end{tabular}

de humedad del grano LRB-106A 1 xLRB-25 $(0,03)$ y LRB-102A 1 x LRB-63 $(0,03)$, para longitud de panícula LRB-102A $\mathrm{A}_{2}$ x LRB-63 $(0,06)$ y LRB-110A 2 x74CS-5388 $(0,06)$, y para longitud de excerción LRB- $102 \mathrm{~A}_{2} \mathrm{x}$ SC0599-11 E $(0,18)$. Se aprecia que los valores más altos de ACE correspondieron en mayor proporción a los híbridos en los que intervienen las líneas hembras LRB$110 \mathrm{~A}_{2}$, LRB-102A 1 y LRB-102A 2 .

Aunque en estos resultados. se observó una mayor proporción en los mejores valores de ACG y ACE para los genotipos que presentan el citoplasma $\mathrm{A}_{2}$, no se encontró una relación entre el tipo de citoplasma y la ACG y ACE.
Esto está de acuerdo a lo señalado por Murty (1986) al estudiar la ACG y ACE en tres tipos de citoplasma $\left(\mathrm{A}_{1}\right.$, $\mathrm{A}_{2}$ y $\mathrm{A}_{3}$ ) en sorgo, en las características de rendimiento de grano, días a floración, altura de planta y producción de materia seca. Por otra parte Chrisi y Miller (1987) al estudiar la heterosis y aptitud combinatoria en un grupo de híbridos $\mathrm{A}_{2}$, encontró que los híbridos $\mathrm{A}_{1}$ consistentemente rindieron más que los híbridos $A_{2}$, excepto para dos de éstos en los que se obtuvieron resultados similares a los de $\mathrm{A}_{1}$. Esto no estáde acuerdo con los resultados obtenidos en este estudio, en donde los híbridos con los dos tipos de citoplasma $\left(\mathrm{A}_{1} \mathrm{y}_{2}\right)$, rindieron de manera similar (Cuadro 2). Hay que 
considerar que la diferencia se puede deber a que Chrisi y Miller trabajaron híbridos de diferente constitución genética y no con híbridos isogénicos como en este trabajo. Obviamente son más confiables los resultados obtenidos de esta última manera.

Las estimaciones de los componentes de varianza para la ACG y ACE para todas las características estudiadas, se encuentran en el Cuadro 5. Una estimación de la importancia relativa de los efectos genéticos aditivos y no aditivos puede ser obtenida por medio de la relación de los componentes generales por la de los efectos específicos. Considerando esto para el caso de rendimiento de grano, los efectos genéticos no aditivos fueron más importantes que los efectos aditivos. En tanto que para días a floración, altura de planta, porcentaje de humedad del grano, longitud de panoja y longitud de excerción fueron más importantes los efectos genéticos aditivos.

Por otra parte los valores de los componentes de varianza para los caracteres de días a floración, altura de planta y longitud de panoja, fueron mayores entre machos que entre las hembras, indicando con esto que existe una mayor variabilidad genética para estos caracteres entre machos. Lo contrario ocurre para las características de rendimiento de grano, \% de humedad del grano a la cosecha y longitud de exerción, donde la varianza entre machos fue menor que la varianza entre hembras. Era de esperarse que existieran una mayor diversidad genética, entre los machos utilizados en este estudio, ya que provienen de diferentes origenes y razas y tienen diferencias en los genes nucleares y citoplásmicos. Mientras que las líneas progenitoras hembras aunque cuenten con diferente constitución nuclear, comparten solo dos tipos de citoplasma.

Se han liberado algunas líneas parentales con el citoplasma $\mathrm{A}_{2}$, para utilizarse en la formación de híbridos comerciales tales como A2 Tx - 2753 (Schertz, 1977), A 2 TAM-428, A 2 Tx-624 y A 2 Tx-2788 (Schertz, Rosenow y Sotomayor, 1981); $\mathrm{A}_{2}$ BTx-632 (Miller, 1986) y $\mathrm{A}_{2} \mathrm{BTx}$ 636 y A ${ }_{2}$ BTx-637 (Miller, Dusek y Prihoda, 1992). Los resultados de ACG y ACE de este estudio están de acuerdo con estas liberaciones, indicando que cuando se toman en cuenta los valores de aptitud combinatoria (ACG y ACE), en híbridos isogénicos para comparar dos tipos de citoplasma $\left(\mathrm{A}_{1} \mathrm{y}_{2}\right)$, no se encontró ninguna desventaja en la utilización del sistema $\mathrm{A}_{2}$ en lugar del $\mathrm{A}_{1}$ para la formación de híbridos comerciales.

Cuadro 5. Componentes de varianza para la aptitud combinatoria general y específica y sus interacciones con ambientes.

\begin{tabular}{lcccccc}
\hline $\begin{array}{l}\text { Causas de } \\
\text { Variación }\end{array}$ & $\begin{array}{c}\text { Rendimiento } \\
\text { kg/ha }\end{array}$ & $\begin{array}{c}\text { Días a } \\
\text { Flor }\end{array}$ & $\begin{array}{c}\text { Altura } \\
\text { Planta }\end{array}$ & $\begin{array}{c}\text { Humedad } \\
\text { Grano }\end{array}$ & $\begin{array}{c}\text { Longitud } \\
\text { Panoja }\end{array}$ & Excerción \\
\hline Ambientes & & & & & & \\
Repeticiones(A) & & & & & & \\
Machos & $26096,5^{* *}$ & $5,48^{* *}$ & $17,0^{* *}$ & $10,8^{* *}$ & $3,54^{* *}$ & $0,80^{* *}$ \\
M x A & $31209,1^{* *}$ & $0,77^{* *}$ & $0,8^{* *}$ & $516,2^{* *}$ & $0,56^{* *}$ & $0,27^{* *}$ \\
Error(a) & $39440,7^{*}$ & $1,01^{* *}$ & $1,9^{*}$ & $132,7 \mathrm{NS}$ & $0,56 \mathrm{NS}$ & $0,19 \mathrm{NS}$ \\
Hembras & $44161,0^{*}$ & $0,80^{* *}$ & $1,4^{* *}$ & $65,5^{* *}$ & $0,35^{* *}$ & $1,70^{* *}$ \\
H x A & $41132,1^{* *}$ & $0,11^{* *}$ & $5,6^{*}$ & $1697,1^{* *}$ & $0,72 \mathrm{NS}$ & $5,06^{* *}$ \\
H x M & $92645,0^{* *}$ & $0,90^{* *}$ & $3,0^{* *}$ & $293,0 \mathrm{NS}$ & $0,012 \mathrm{NS}$ & $0,19 \mathrm{NS}$ \\
H x M x A & $163523,3^{* *}$ & $3,16^{* *}$ & $2,4^{* *}$ & $827,8 \mathrm{NS}$ & $0,007^{* *}$ & $3,5^{* *}$ \\
Error B & 647649,0 & 5,344 & 81,08 & 20289,2 & 16,43 & 29,93 \\
\hline
\end{tabular}

Total 


\section{CONCLUSIONES}

1. Aunque en general los resultados indicaron que los mejores valores de ACG yACE correspondieron a genotipos con el sistema $\mathrm{A}_{2}$, comparando los valores de los pares de híbridos isogénicos entre sí $\left(\mathrm{A}_{1}\right.$ y $\left.\mathrm{A}_{2}\right)$, no se encontró ninguna relación entre aptitud combinatoria y el tipo de citoplasma.

2. Los resultados sugieren que es factible utilizar el sistema de androesterilidad génico-citoplásmico $\mathrm{A}_{2}$, en la formación de híbridos a nivel comercial como una alternativa a la utilización del sistema $\mathrm{A}_{1}$.

\section{LITERATURA CITADA}

BEILS, G.M.; ATKINS, R.E. 1967. Estimates of general and specific combining ability in $\mathrm{F}_{1}$ hybrids for grain yield and its components in grain sorghum, Sorghum vulgare Pers. Crop Sci. 7:225-228.

CHRISE, M.; MILLER, F.R. 1987. Comparison of $\mathrm{F}_{1}$ hybrids in $A_{1}$ and $A_{2}$ cytoplasm across locations. Sorghum newsletter 30:23.

GANGA KRISHAN, A.; BORIKAR, S. 1989. Genetic relationship between some cytoplasmic male sterility systems in sorghum. Euphytica. 42:259-264.

HANNA, W.W. 1989. Characteristics and stability of a new cytoplasmic nuclear male sterile source in pearl millet. Crop Sci. 29: 1457-1459.

LEE, D.M.; ALBERTSEN, N.; RASMUSSON, D.C. 1987. Cytoplasmic effects on agronomic and malting quality traits in barley. Crop Sci. 27:669-673.

MILLER, F.R. 1986. Registration of se ven sorghum A and B line inbreds. Crop Sci. 26: 216-217.

, DUSEK, T.F.; PRIHODA, K.L. 1992. Registration of $A_{2} / B_{2}$ Tx636 and $A_{2} / B_{2}$ Tx637 sorghum. Crop Sci. 32: 511-512.
MURTY, U.R. 1986. Cytoplasmic effects on heterosis and combining ability in grain sorghum. Sorghum Newsletter 29:76.

PROCEEDINGS OF THE PLANT BREEDING METHODS AND APPROACHES IN SORGHUM WORKSHOP FOR LA TIN AMERICA. (1983, El Batan, Mexico) 1983. Cytoplasms and potential for reduction of genetic vulnerability in sorghum. Publicado por Schertz, K.F. Ed. INTSORMIL. El Batan, México. pp 114-118.

ROSS, W.M., KOFOID, K.D. 1979. Effects of non-milo cytoplasms on the agronomic perforrnance or sorghum. CropSci. 19:267-270.

SCHERTZ, K.F..1977. Registration of $\mathrm{A}_{2} \mathrm{Tx} 2753$ and $\mathrm{B}_{2} \mathrm{~T} \times 2753$ sorghum germoplasm. Crop Sci. 17:988.

, ROSENOW, D.T.; SOTOMAYOR RIOS, A.

1981. Registration of three pairs (A and B) of sorghum germoplasms with $\mathrm{A}_{2}$ citoplasmic genetic sterility system. Crop Sci. 21: 148.

RITCHEY, J.M. 1978. Cytoplasmic-genic male sterility systems in sorghum. Crop Sci. 18: 890-893.

STEPHENS, J.C.; HOLLAND, R.F. 1954. Cytoplasmic male sterility for hybrids sorghum seed production. Agron. J. 46: $20-23$.

WILLIAMS, A.H.; RODRIGUEZ, H.R. 1994. Comparative performance of sorghums in $\mathrm{A}_{1}$ and $\mathrm{A}_{2}$ cytoplasms. II. Yield and agronomic characteristics. Cereal Research Communications. En prensa.

WORSTELL, J.V.; KIDD, H.J.; SCHERTZ, K.F. 1984. Relationships . among male-sterility inducing cytoplasms of sorghum. Crop. Sci. 24: 186-189. 